\title{
1 Percutaneous repair of acute Achilles Tendon ruptures. The Maffulli procedure
}

\author{
Nicola Maffulli, Francesco Oliva, Mario Ronga
}

\section{Introduction}

The Achilles tendon (AT) is the strongest tendon in the human body with a tensile strength in the order of 50 $100 \mathrm{~N} / \mathrm{mm}(1,2)$. It is formed from the joining of the two tendons of soleus (dorsally) and gastrocnemius (ventrally). Despite its strength, the AT is the most frequently ruptured tendon in the body. Tendon fibres begin to disrupt after a length increase of 3-4\% and rupture after an increase of $8 \%$. Ruptures usually occur between 2-6 $\mathrm{cm}$ of its insertion into the superior surface of the calcaneus, a relatively hypovascular area $(3,4)$. The major blood supply to tendons is from the mesotendon, and the largest supply is from the anterior mesentery (5). The tendon is at the greatest risk of rupture when the it is obliquely loaded, the muscle is contracting maximally, and tendon length is short. ${ }^{6}$ This usually occurs as a result of pushing off with the foot against resistance, and occurs most frequently in males in their fourth decade.

The aetiopathogenesis of AT rupture is unknown, but histological evidence of failed healing response is relatively common. McMaster suggested that normal tendons do not rupture (6). Tallon et al. (7) showed that both tendinopathic and ruptured tendons had a greater degree of histological evidence of failed healing response compared with normal tendons, and that the degree of degeneration in the ruptured group was statistically greater than in the tendinopathic group. AT rupture has also been associated with steroid use, rheumatoid arthritis, renal transplantation, and quinolone antibiotic use (8-11).

Non-operative management of AT rupture was described as early as 1575 by Ambroise Pare who treated these injuries with bandages but noted a poor outcome. Immobilization with an equinus cast has a reported rerupture rate of $17 \%$ (13), and will result in significant loss of strength and power in the calf muscle (14). Open surgical management of patients with ruptured ATs allows accurate apposition of the ruptured tendon ends, earlier motion, has a low risk of re-rupture, but is associated with a significant rate of wound healing problems. Advocates of minimally invasive AT surgery cite faster recovery times, shorter hospital stays, and improved functional outcomes as the principal reasons for adopting these new approaches when compared to traditional open techniques. Open procedures on the AT can lead to difficulty with wound healing because of the tenuous blood supply, and thus increased chance of wound breakdown and infection. Moreover, the broad exposure given by open procedures may case extensive iatrogenic disruption of the subcutaneous tissues and paratenon, increasing the potential for peritendinous adhesions. Critics have raised questions about increased percentage of complications (i.e. sural nerve damage) (15). Nevertheless, percutaneous techniques allow a secure repair to be achieved. Because of the perceived greater strength of the suture and despite the risks of wound complications, open repair has traditionally considered the treatment method of choice for young fit patients wishing to return to sporting activities (16).

Bradley and Tibone (16) reported a higher risk of rerupture in percutaneous repairs compared with open repair. Lim et al more recently, however, in a prospective multicentre randomised controlled study comparing open and percutaneous repair techniques reported a higher rate of re-rupture in patients treated by an open technique $(6 \%$ vs $3 \%$, using a percutaneous technique). The difference, however, was not statistically significant. Bradley and Tibone (16) compared 15 patients treated with a gastrocsoleus fascial graft and 12 patients treated using a percutaneous technique. Strength, power and endurance measurements of both groups showed no statistical difference. Two of 12 (13\%) percutaneous repairs re-ruptured up to a follow-up of 1.8 years, compared with none in the open repair group (follow-up 4.6 years). They recommended percutaneous repair in the recreational athlete and open repair in the competetive athlete. Martinelli 18 reported 30 cases of percutaneous repair of ATs in which all athletes returned to preinjury levels of sport by 150 days post-injury.

Wound healing problems associated with open repair can be reduced by using percutaneous techniques, and the incidence of adhesion of the skin to the under- 
lying tendon, as can occur in open repair, is also lower with percutaneous techniques $(13,17)$.

Kauranen and Leppilahti (19) reviewed the motor performance of 90 patients following operative repair (mean of 3.1 years post surgery) of a ruptured AT. They observed the performance of the unloaded lower extremity, and compared the operated limbs with the unoperated side, and to age and gender-matched control subjects. They found no statistical difference between any of the groups, and concluded that the motor performance of the unloaded lower limb had fully recovered in the tested parameters.

Several percutaneous repair techniques have been described (13, 20-22). Ma and Griffith described a technique of percutaneous repair of the AT in 18 patients using stab incisions over the tendon (20). The suture was passed through stab incisions and crisscrossed through the tendon.

They reported some patients with sensory disturbance and one case of sural nerve entrapment. Hockenbury and John noted sural nerve entrapment in 3 of 5 cases treated using a percutaneous technique in cadaveric specimens with divided ATs (5).

Gorschewsky et al. described a technique using barbed suture wires passed from proximal to distal to pull the retracted proximal stump distally and approximate the ruptured tendon ends. Fibrin glue was then applied to the repair. The wires were removed at 3 weeks. At one year follow-up in 20 patients there was one re-rupture and no other complications (21).

Webb and Bannister described a new percutaneous technique that reduced the potential risk to the sural nerve by placing the most proximal of the incisions to the medial side, away from the nerve (13). We described a percutaneous technique of repair of the ruptured ATs similar to that described by Webb and Bannister (13), but using a more secure suture configuration.

Recently, several authors reported on the Achillon mini-incision technique, comparing the basic mechanical properties of the tendon suture performed using the Achillon method with those of the Kessler method, and assesses whether the strength of the repair was related to tendon diameter. The Achillon repair had comparable tensile strength to the Kessler repair.

When compared to the Achillon repair our technique (27) is cheaper, and allows a stronger repair, as it allows to use a greater number of suture strands (eight) for the repair of the AT.

\section{Pre-operative preparation}

The diagnosis is generally made clinically. Patients will often report that thay felt as though they had been struck at the back of the heel and may have heard a snapping sound. They are usually unable to weight bear on the affected limb because of pain and/or weakness. If examined shortly after the injury, there is usually a palpable defect in the AT. If some time has elapsed since the rupture, the diagnosis can be more difficult, as the gap fills in with oedema and palpation is unreliable. Various tests can be employed to aid diagnosis (23-25). The calf squeeze test involves the examiner gently squeezing the patient's calf muscles with the palm of their hand (23). If the AT is intact, the ankle plantar flexes. If the AT is torn, the ankle remains still, or only minimally plantar flexes. The Matles test can also be used (24). It involves flexing the patient's knee to 90 degrees with the patient prone. If the foot falls into neutral or dorsiflexion, a ruptured AT is diagnosed. Both tests are performed on the injured and uninjured sides for comparison. The needle test is accurate, but uncomfortable for the patient and, therefore, we use it infrequently. We do not routinely use imaging to diagnose a ruptured AT.

The neurovascular status of the limb is assessed, in particular the sensation over the distribution of the sural nerve, and documented. The risks of surgery, including sural nerve injury, re-rupture, wound infection and breakdown, are discussed with the patient when informed consent is obtained.

\section{Surgical procedure}

The patient is positioned prone (27). Areas $4-6 \mathrm{~cm}$ proximal and distal to the palpable tendon defect and the skin over the defect are infiltrated with $20 \mathrm{~mL}$ of $1 \%$ Lignocaine. $10 \mathrm{~mL}$ of Chirocaine $0.5 \%$ are infiltrated deep to the tendon defect. A calf tourniquet, skin preparation and steridrapes are applied.

A $1 \mathrm{~cm}$ transverse incision is made over the defect using a size 11 blade. Four longitudinal stab incisions are made lateral and medial to the tendon $6 \mathrm{~cm}$ proximal to the palpable defect. Two further longitudinal incisions on either side of the tendon are made $4-6 \mathrm{~cm}$ distal to the palpable defect. Forceps are then used to mobilise the tendon from beneath the subcutaneous tissues. A $9 \mathrm{~cm}$ Mayo needle (BL059N, \#B00 round point spring eye, B Braun, Aesculap, Tuttlingen, Germany) is threaded with 2 double loops of Number 1 Maxon (Tyco Healthcare, Norwalk, CT, USA), and this is passed transversely between the proximal stab incisions through the bulk of the tendon (Figure 1). The bulk of the tendon is surprisingly superficial. The loose ends of the suture are held with a clip. In turn, each of the ends is then passed distally from just proximal to the transverse Maxon passage through the 


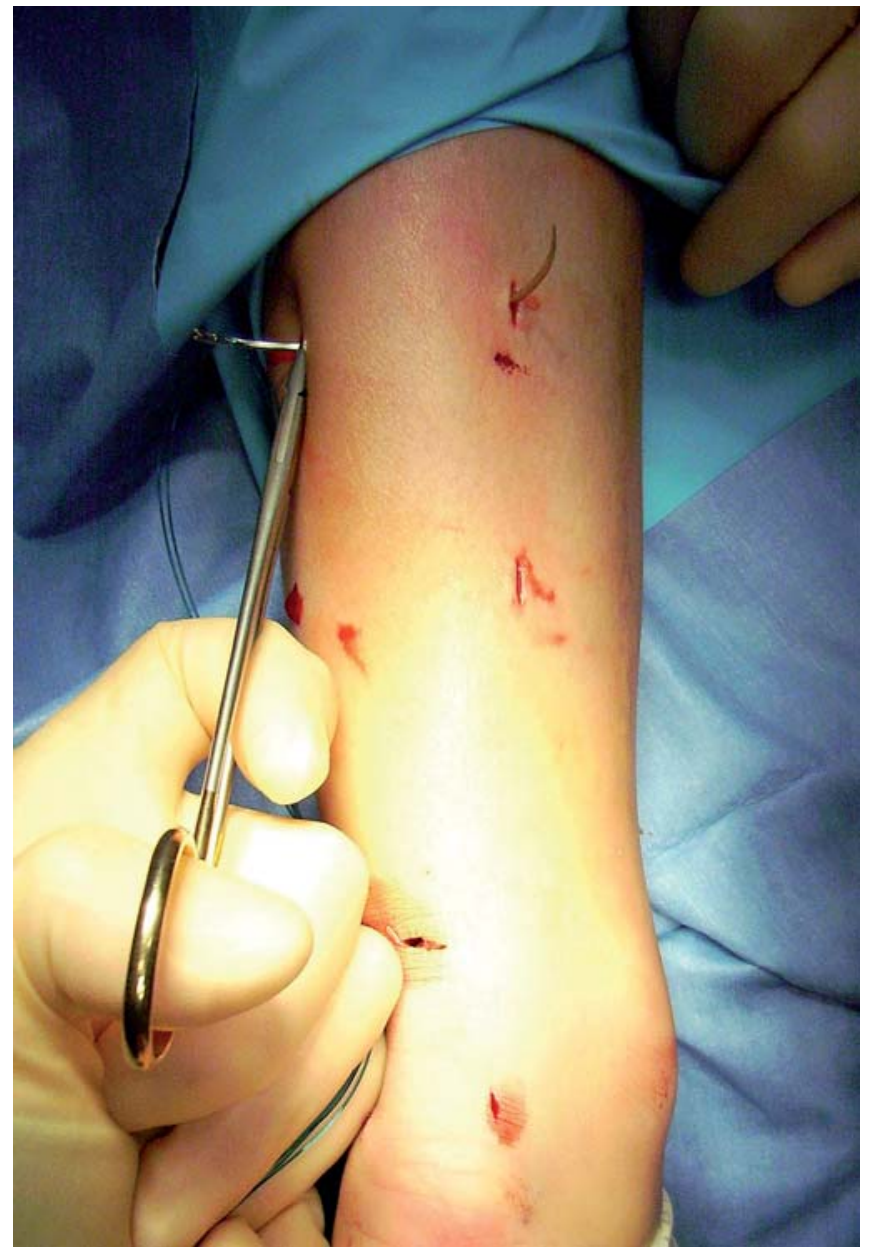

Figure 1 A $9 \mathrm{~cm}$ Mayo needle (BL059N, \#B00 round point spring eye, B Braun, Aesculap, Tuttlingen, Germany) is threaded with 2 double loops of Number 1 Maxon (Tyco Healthcare, Norwalk, CT, USA), and this is passed transversely between the proximal stab incisions through the bulk of the tendon.

bulk of the tendon to pass out of the diagonally opposing stab incision. A subsequent diagonal pass is then made to the transverse incision over the ruptured tendon. To prevent entanglement, both ends of the Maxon are held in separate clips. This suture is then tested for security by pulling with both ends of the Maxon distally. Another double loop of Maxon is then passed between the distal stabs incisions through the tendon (Figure 2), and in turn through the tendon and out of the transverse incision starting distal to the transverse passage in a half Kessler configuration (Figure 3). The ankle is held in full plantar flexion, and in turn opposing ends of the Maxon thread are tied together with a double throw knot, and then three further throws before being buried using the forceps. A clip is used to hold the first throw of the lateral side to maintain the tension of the suture.

A subcuticular Biosyn suture 3.0 (Tyco Healthcare) is used to close the transverse incision, and Steri-strips

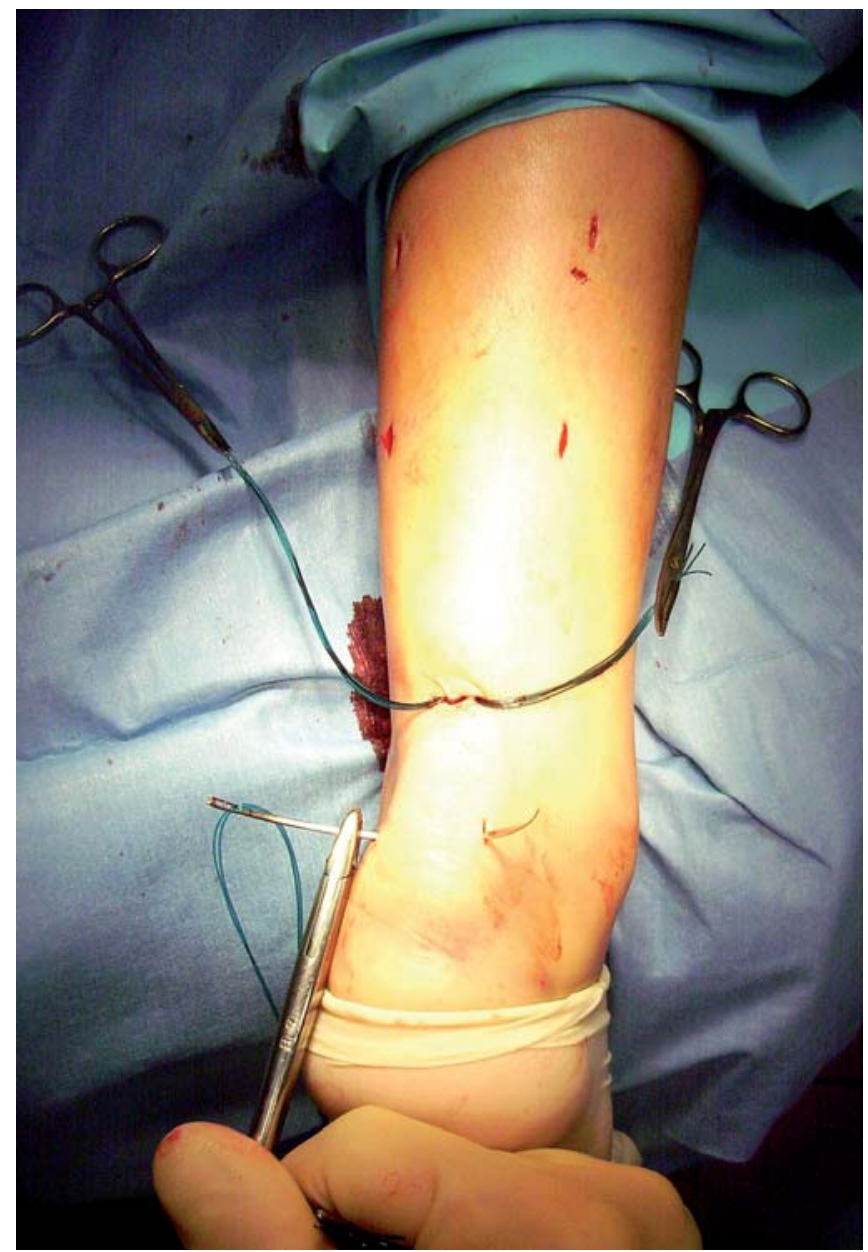

Figure 2 Another double loop of Maxon is then passed between the distal stabs incisions through the tendon.

(3M Health Care, St Paul, MN, USA) are applied to the stab incisions. Finally, a Mepore dressing (Molnlycke Health Care, Gothenburg, Sweden) is applied, and a bivalved removable scotch cast in full plantar flexion is applied being held in place with Velcro straps.

The patient is allowed home on the day of surgery, and fully weight bears as able in the cast in full plantar flexion. At 2 weeks, the wounds are inspected, and the back shell is removed allowing proprioception, plantar flexion, inversion and eversion exercises. The front shell remains in place for 6 weeks to prevent forced inadvertent dorsiflexion of the ankle.

\section{Possible complications}

Complications can be early, intermediate or late, and are outlined in Table 1.

Early possible post-operative complications are 


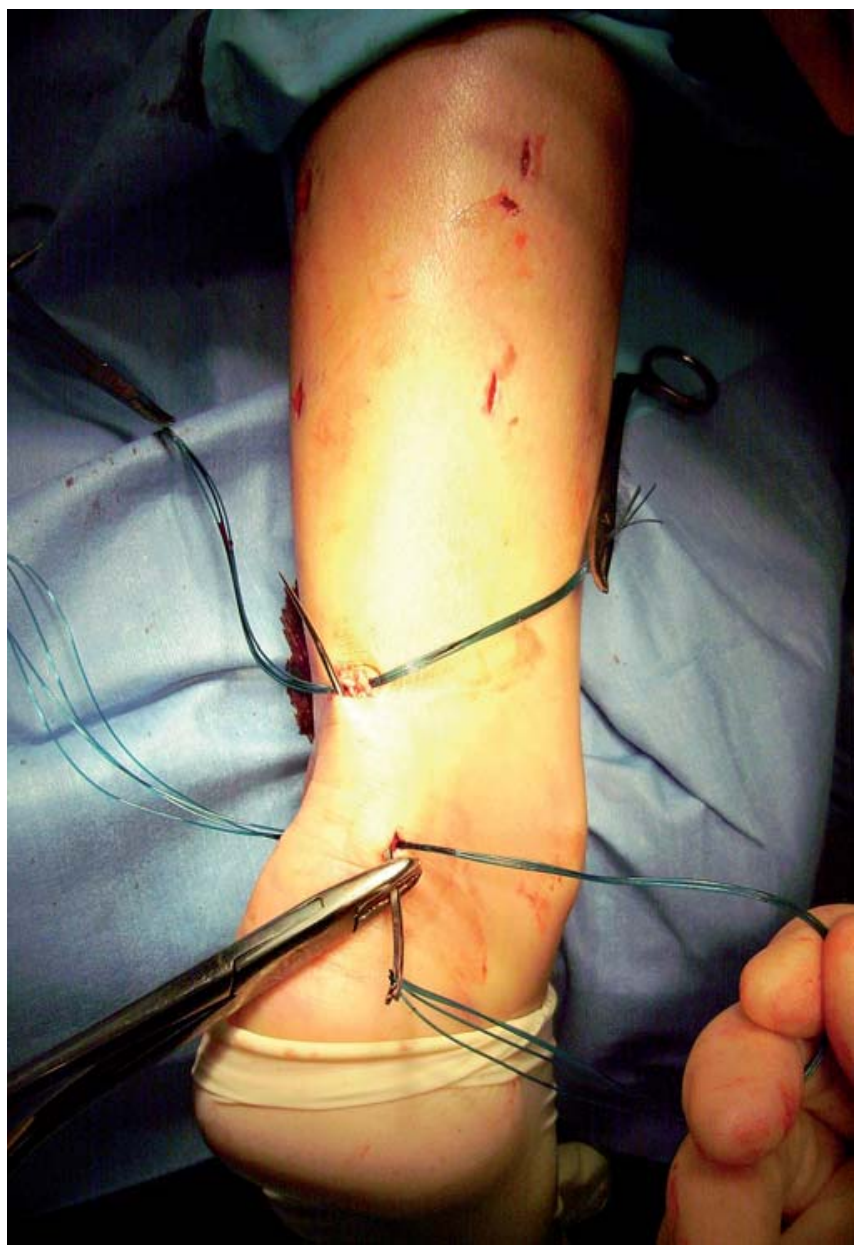

Figure 3 The double loop of Maxon is passed in turn through the tendon and out of the transverse incision starting distal to the transverse passage.

sural nerve damage and haematoma formation. The positioning of the incisions and the configuration of the stitch reduces the risk of damage to the sural nerve. The risk of haematoma formation is reduced as the procedure is carried out without tourniquet so that the surgeon will be able to deal with any bleeding at the time of operation.

Intermediate superficial and deep wound infections can occur. Open repair is associated with a significant risk of wound breakdown. Percutaneous repair reduces this risk.

The most important late complication is re-rupture. In a review of reported rates of re-rupture from conservatively treated AT ruptures, Webb and Bannister reported a median $17 \%$ risk (13). They reported a risk of rerupture-with operatively treated AT rupture of $2.7 \%$, from a review of the literature. Using a percutaneous technique, Webb and Bannister did not have a re-rupture at a median follow-up of 35 months in 27 patients. Bradley and Tibone reported a re-rupture
Table 1 Complications which can occur following a ruptured AT

\begin{tabular}{ll}
\hline Early (peri-operative) & $\begin{array}{l}\text { Sural nerve damage } \\
\text { Haematoma }\end{array}$ \\
Intermediate ( $<6$ weeks) & $\begin{array}{l}\text { Infection } \\
\text { Wound healing complications }\end{array}$ \\
Late (6 weeks to 6 months) & Re-rupture of tendon
\end{tabular}

Table 2 Key points of rupture and repair.

Patient selection
Prone position
Local anaesthesia
Incisions made to avoid sural nerve
Four and if necessary eight strand repair
Splintage to protect repair for total of 6 weeks

rate of $13 \%$ (2/12 patients) at a follow-up of 1.8 years (16). However, re-rupture rates of $6 \%$ have been reported for percutaneous repairs.

In conclusion, the nascent literature on minimally invasive AT repair is far from universally supportive. Most published series on these surgical approaches are first reports by originators of particular techniques. Randomized controlled trials are required to address the issue of the comparison between open versus minimally invasive AT surgery. In our hands, minimally invasive surgery has provided similar results to those obtained with open surgery, providing decreased perioperative morbidity, decreased duration of hospital stay, and reduced costs.

\section{References}

1. Viidik A. Tensile strength properties of Achilles tendon systems in trained and untrained rabbits. Acta Orthop Scand 10: 261-272, 1962.

2. Williams PL, Warwick R, Dyson M, Bannister LH. Grays Anatomy. 37th Ed. Churchill Livingstone 1989.

3. Williams IF. Cellular and biochemical composition of healing tendons in Ligament injuries and their treatment. $9 \mathrm{Ed}$ Jenkins DHR). London. Chapman \& Hall 3: 43-47, 1985.

4. Langergren C, Lindholm A. Vascular distribution in the Achilles tendon. Acta Chir Scand 116: 491-495, 1958.

5. S. Terry Canale. Campbell's Operative Orthopaedics. 9th Ed.

6. McMaster PE. Tendon and muscle ruptures: Clinical and experimental studies on the causes and location of subcutaneous ruptures. J Bone Joint Surg 15: 705-722, 1933.

7. Tallon C, Maffulli N, Ewen SW. Ruptured Achilles tendons are significantly more degenerated than tendinopathic tendons. Med Sci Sports Exerc 33(12): 1983-90, 2001.

8. Haines JF. Bilateral rupture of the Achilles tendon in patients on steroid therapy. Ann Rheum Dis 42(6): 652-4, 1983.

9. Rask MA. Achilles tendon rupture owing to Rheumatoid disease. JAMA 239: 435-6, 1978. 
10. Hestin D, Mainard D, Pere P, Bellou A, Renoult E, Cao Huu T, Chaniliau J, Kessler M. Spontaneous bilateral rupture of the Achilles tendons in a renal transplant recipient. Nephron 65: 491-2, 1993.

11. Poon CC, Sundaram NA. Spontaneous bilateral Achilles tendon rupture associated with Ciprofloxacin. Med J Aust 166(12): 665, 1997

12. Carlstedt CA. Mechanical and chemical factors in tendon healing : Effects of indomethacin and surgery in the rabbit. Acta Orthop Scand 58 (Suppl 224): 1-75, 1987.

13. Webb JM, Bannister GC. Percutaneous repair of the ruptured tendo Achillis. J Bone Joint Surg Br 81(5): 877-80, 1999.

14. Haggmark T, Liedberg H, Eriksson E, Wredmark T. Calf muscle atrophy and muscle function after nonoperative vs operative treatment of tendon ruptures. Clin Orthop 9: 160$164,1986$.

15. Hockenbury RT, Johns JC. A biomechanical in vitro comparison of open versus percutaneous repair of tendon Achilles. Foot Ankle 11(2): 67-72, 1990.

16. Bradley JP, Tibone JE. Percutaneous and open surgical repairs of Achilles tendon ruptures. A comparative study. Am J Sports Med 18: 188-195, 1990.

17. Lim J, Dalal R, Waseem M. Percutaneous vs. open repair of the ruptured Achilles tendon - a prospective randomized controlled study. Foot Ankle Int 22(7): 559-68, 2001.

18. Martinelli B. Percutaneous repair of the Achilles tendon in athletes. Bull Hosp Jt Dis 59(3): 149-52, 2000.
19. Kauranen KJ, Leppilahti JI. Motor performance of the foot after Achilles rupture repair. Int J Sports Med 22(2): 154-8, 2001.

20. Ma GW, Griffith TG. Percutaneous repair of acute closed ruptured achilles tendon: a new technique. Clin Orthop (128): 247-55, 1977.

21. Gorschewsky O, Vogel U, Schweizer A, van Laar B. Percutaneous tenodesis of the Achilles tendon. A new surgical method for the treatment of acute Achilles tendon rupture through percutaneous tenodesis. Injury 30(5): 315-21, 1999.

22. Cretnik A, Zlajpah L, Smrkolj V, Kosanovic M. The strength of percutaneous methods of repair of the Achilles tendon: a biomechanical study. Med Sci Sports Exerc 32(1):16-20, 2000.

23. Simmonds FA. The diagnosis of the ruptured Achilles tendon. The Practitioner 179: 56-58, 1957.

24. Matles AL. Rupture of the tendo Achilles. Another diagnostic test. Bull Hosp Joint Dis 1975, 36: 48-51, 1975.

25. O Brien T. The needle test for complete rupture of the Achilles tendon. J Bone Joint Surg (A) 66: 1099-1101, 1984.

26. Webb J, Moorjani N, Radford M. Anatomy of the sural nerve and its relation to the Achilles tendon. Foot Ankle Int 21(6): 475-7, 2000.

27. Carmont MR, Maffulli N. Modified percutaneous repair of ruptured Achilles tendon. Knee Surg Sports Traumatol Arthrosc 16(2):199-203, 2008. 